\title{
Ellipsoidal Set-Membership State Estimation for Multi-Output Systems with Interval Uncertainties
}

\author{
S. Ben Chabane, C. Stoica Maniu, T. Alamo, E. F. Camacho, D. Dumur
}

\begin{abstract}
This paper presents a new online ellipsoidal guaranteed set-membership state estimation approach for MultiOutput linear systems with interval uncertainties, bounded perturbations and measurement noises. The ellipsoidal set containing the real state of the system is computed at each sample time. This approach consists in online minimizing the radius of the ellipsoidal state estimation set by solving a Linear Matrix Inequality optimization problem. Interval uncertainties are considered in both the evolution matrix and the observation matrix. An illustrative example is analyzed in order to show the advantages of the proposed approach.
\end{abstract}

Index Terms - set-membership state estimation, ellipsoidal set, interval uncertainty, Linear Matrix Inequality.

\section{INTRODUCTION}

Knowledge of the system state plays a very important role in systems monitoring and control. However, in many cases the full state is not directly measurable and an estimation of the system state is required. In addition, the presence of disturbances and measurement noises makes this estimation more difficult. Using the available online measurements and a dynamic model of the process, the idea is to compute the best estimate of the state variables at the current time, despite the possible perturbations and measurement noises.

More often, the state estimation problems are solved by implementing a stochastic approach based on a probabilistic description of the perturbations and measurement noises [1]. This requires to assume that the individual perturbations are realizations of random variables characterized statistically by their average, covariance, probability density etc. However, sometimes the probabilistic assumptions are difficult to verify. Thus, it may be more realistic to assume that the errors belong to compact bounded sets. This corresponds to the deterministic approach or the set-membership estimation [2], [3], [4], [5], where perturbations are considered unknown but bounded. In set-membership estimation approaches, the evolution of system states at each time instant is not described by a point in the state-space but by a set consistent with the

The authors acknowledge MEyC Spain (contract DPI2016-76493-C3-1R), the European Research Council and the UE ERDF (Advanced Grant OCONTSOLAR, Project ID: 789051).

S. Ben Chabane is with Signal Processing Department, Control Team, Parrot Drones, 174-178 Quai de Jemmapes, 75010 Paris, France (e-mail: sofiane.benchabanelparrot.com).

C. Stoica Maniu and D. Dumur are with Université Paris-Saclay, CNRS, CentraleSupélec, Laboratoire des signaux et systèmes, 91190, Gif-sur-Yvette, France. (e-mail: \{cristina.stoica; didier.dumur\}@l2s.centralesupelec.fr).

T. Alamo and E.F. Camacho are with Department of Ingeniería de Sistemas y Automática, Universidad de Sevilla, Camino de los Descubrimientos, 41092 Sevilla, Spain (e-mail: talamo@us.es, eduardodesi.us.es) measurements, the perturbations, the uncertainties and the noise. This set guarantees to contain the real state. While their use was severely restricted in the 80 s due to the low capacity of available computers, these approaches have been widely used over the last two decades by many researchers [6], [7], [8], [9].

Several domain representations are used to bound the set of all possible states of the system consistent with the measurements and perturbations, such as polytopes [10], parallelotopes [7], zonotopes [11], [12], [13], [14], [15], [16] and ellipsoids [17], [18], [19], [20]. Ellipsoidal estimation has increased the attention among researchers in the last decades. Indeed, ellipsoids are widely used due to the simplicity of their formulation and resulting estimation stability properties [21]. This topic is considerably covered in [17], which in particular provides an exhaustive solution of the problem for the sum and the geometrical difference of ellipsoids. Less efforts have been devoted to the study of other important operations, such as the union and the intersection, although the construction of external approximations for the intersection of ellipsoids plays a key role for control design in dynamical systems with ellipsoidal constraints. To minimize the size of the estimation ellipsoidal set, two methods are mainly considered. Firstly, the determinant-based criterion is minimized [18], which is equivalent to minimize the volume of the ellipsoidal set. Secondly, the minimization of the trace criterion, which is equivalent to minimize the sum of squares of the half length of the axes of the ellipsoid, is considered in the literature [18]. These two methods offer low complexity suitable for online implementation, but with a loss of accuracy compared to the polytopic estimation [18].

In addition, zonotopes are proposed to obtain a better estimation accuracy than ellipsoids. To minimize the size of the zonotopic estimation, several methods are proposed in the literature. In [12], a method based on the Singular Value Decomposition is used in order to obtain a zonotopic outer approximation of the intersection of the uncertain trajectory and the region which is consistent with the measured output vector. In [13], the minimization of the segments and the volume of the zonotope are used. The segments minimization method is faster but less accurate than the minimization of the volume of the zonotope. In [14], the minimization of the $P$-radius of the zonotope leads to a good tradeoff between the rapidity of the segments minimization and the estimation accuracy of the volume minimization of a zonotope. The $P$-radius minimization zonotopic method [14] is the only existing zonotopic method used for linear systems with interval uncertainties affecting the evolution matrix. 
Several works on interval observers have been developed for these uncertain dynamical systems [22], [23], [24]. The main difficulty arising when dealing with interval observers [25] is the dependency problem, which is an important consequence of the so-called wrapping effect for uncertain systems.

In this context, the aim of this paper is to propose a new online ellipsoidal set-membership estimation approach for linear uncertain systems with bounded perturbations, measurement noises and interval uncertainties on both the evolution matrix and observation matrix. In this approach, the novelty consists in proposing an online ellipsoidal state estimation procedure based on the minimization of the ellipsoid radius which allows to take into account simultaneously bounded perturbations, measurement noises and inverval uncertainties both in the evolution and the observation matrices. This result is based on the use of the S-procedure for quadratic functions [26]. It offers less conservative results than the estimation obtained by the off-line $P$-radius-based zonotopic estimation method [14] due to the minimization of the ellipsoidal radius at each time instant. The ellipsoidal estimation was initially developed for linear systems with bounded perturbations and measurement noises in [27], while considering an interval evolution matrix in [28]. The originality of the present paper is to extend this method to the case of linear time-invariant systems with bounded perturbations, bounded measurement noises and interval uncertainties in both the evolution and observation matrices. Considering structured uncertainties in the observation matrix is a main issue for sensor fault detection and isolation.

This paper is organized as follows. The state estimation problem is formulated in Section II. Section III focuses on the main results of this paper, presenting a new approach for guaranteed ellipsoidal state estimation for linear timeinvariant systems with interval uncertainties. Section IV proposes an illustrative example showing the advantages of the proposed method. Finally, some concluding remarks and perspectives are drawn.

Notations An interval $[a, b]$ is defined by the set $\{x \in \mathbb{R}: a \leq x \leq b\}$. The set of real compact intervals $[a, b]$, with $a, b \in \mathbb{R}$ and $a \leq b$, is denoted by $\mathbb{I}$. The unitary interval $\mathbf{B}$ is defined by the interval $[-1,1]$. A box $\left(\left[a_{1}, b_{1}\right], \ldots,\left[a_{n}, b_{n}\right]\right)^{\top} \subset \mathbb{R}^{n}$ is an interval vector. A unitary box $\mathbf{B}^{n}$ is composed by $n$ unitary intervals. An interval matrix is defined by $[M] \subset \mathbb{I}^{n \times m}$, with $a_{i j} \leq m_{i j} \leq b_{i j}$, $i=1, \ldots, n$, and $j=1, \ldots, m$, with $\operatorname{mid}[M]_{i j}=\frac{a_{i j}+b_{i j}}{2}$ and $\operatorname{rad}[M]_{i j}=\frac{a_{i j}-b_{i j}}{2}$ defining its center and radius, resp. A set $\mathcal{V}_{[M]}$ defines the set of all vertices of the interval matrix $[M]$. A polyhedral set $\mathcal{X}$ in a finite-dimensional euclidean space is given by the set $\left\{x \in \mathbb{R}^{n}: A x \leq b, A \in \mathbb{R}^{m \times n}, b \in \mathbb{R}^{m}\right\}$. $M \succ 0$ denotes a positive definite matrix. A bounded ellipsoid $\mathcal{E}(P, \bar{x}, \rho)$ is defined by $\mathcal{E}(P, \bar{x}, \rho)=\left\{x \in \mathbb{R}^{n_{x}}\right.$ : $\left.(x-\bar{x})^{\top} P(x-\bar{x}) \leq \rho\right\}$, with $P=P^{\top} \succ 0$ its shape matrix, $\bar{x} \in \mathbb{R}^{n_{x}}$ its center and $\rho \in \mathbb{R}_{+}^{*}$ its radius.

\section{Problem Statement}

Consider the linear discrete-time invariant system:

$$
\left\{\begin{array}{l}
x_{k+1}=A_{\delta} x_{k}+E \omega_{k} \\
y_{k}=C_{\delta} x_{k}+F \omega_{k}
\end{array}\right.
$$

where $x_{k} \in \mathbb{R}^{n_{x}}$ is the state vector of the system and $y_{k} \in$ $\mathbb{R}^{n_{y}}$ is the measured output vector at sample time $k$. The vector $\omega_{k} \in \mathbb{R}^{n_{x}+n_{y}}$ contains both of the state perturbations and the measurement perturbations (noise, offset, etc.), which can be non-correlated. It is assumed that the perturbations $\omega_{k}$ are bounded by the unitary box $\mathbf{B}^{n_{x}+n_{y}}$ and the initial state $x_{0}$ is bounded by the ellipsoid: $\mathcal{E}\left(P_{0}, \bar{x}_{0}, \rho_{0}\right)=\{x \in$ $\left.\mathbb{R}^{n_{x}}:\left(x-\bar{x}_{0}\right)^{\top} P_{0}\left(x-\bar{x}_{0}\right) \leq \rho_{0}\right\}$. Matrices $A_{\delta}, C_{\delta}$, $E$, and $F$ have the appropriate dimensions, with the pair $\left(C_{\delta}, A_{\delta}\right)$ detectable (see [29] for the detectability of linear time invariant systems with interval plants). An additional assumption is to consider a quadratically stable interval matrix $[A]$ for a common quadratic Lyapunov function. $A_{\delta}$ and $C_{\delta}$ are constant unknown matrices belonging to interval matrices $[A]$ and $[C]$, which permits to structure the uncertainties $\Delta A_{\delta}$ and $\Delta C_{\delta}$ as follows:

$$
\left\{\begin{array}{l}
A_{\delta}=\operatorname{mid}[A]+\Delta A_{\delta} \\
C_{\delta}=\operatorname{mid}[C]+\Delta C_{\delta}
\end{array}\right.
$$

If $n_{A_{\delta}}$ (resp. $n_{C_{\delta}}$ ) is the number of the uncertain terms $\delta_{i} \in$ $\mathbf{B}\left(\right.$ resp. $\delta_{j} \in \mathbf{B}$ ) of $\Delta A_{\delta}$ (resp. $\Delta C_{\delta}$ ), with $i=1, \ldots, n_{A_{\delta}}$ (resp. $j=1, \ldots, n_{C_{\delta}}$ ), then the uncertain part $\Delta A_{\delta}$ (resp. $\Delta C_{\delta}$ ) can be decomposed into elementary parts:

$$
\Delta A_{\delta}=\sum_{i=1}^{n_{A_{\delta}}} A_{\delta_{i}} \delta_{i} \quad\left(\text { resp. } \Delta C_{\delta}=\sum_{j=1}^{n_{C_{\delta}}} C_{\delta_{j}} \delta_{j}\right) .
$$

The attentive reader can notice that $n_{\delta}=n_{A_{\delta}}+n_{C_{\delta}}$.

At time $k>0$, consider an ellipsoid $\mathcal{E}\left(P, \bar{x}_{k}, \rho_{k}\right)$ that contains the real system state $x_{k}$, with $\bar{x}_{k}$ the nominal estimated set. The aim of this paper is to provide at time $k+1$ an optimal ellipsoidal estimation $\mathcal{E}\left(P, \bar{x}_{k+1}, \rho_{k+1}\right)$ that guarantees to contain the state $x_{k+1}$.

\section{MAIN RESUlts}

This section focuses on the construction of the ellipsoidal guaranteed estimation set containing the real state of the system (1). Suppose that, at time $k$, the ellipsoidal set $\mathcal{E}\left(P, \bar{x}_{k}, \rho_{k}\right)$ is an outer bound of the system state. Suppose also that a measured output $y_{k}$ is obtained at sample time $k$. Based on the minimization of the radius of the ellipsoidal estimation set at each sample time $k$, an outer bound of the online ellipsoidal state estimation is computed here, in the general context of the uncertain system (1).

Knowing that $\omega_{k} \in \mathbf{B}^{n_{x}+n_{y}}$, it is possible to take into account its structure to avoid the vertex enumeration problem. Therefore, the element-wise formulation of $\omega_{k}=$ $\left[\omega_{k_{1}} \omega_{k_{2}} \ldots \omega_{k_{n_{x}+n_{y}}}\right]^{\top} \in \mathbf{B}^{n_{x}+n_{y}}$ allows writting $\left|\omega_{k}\right| \leq$ 1 and $\omega_{k}^{\top} \omega_{k} \leq 1$. Denote by $e_{i}, i=1, \ldots, n_{n_{x}+n_{y}}$, the columns of the following identity matrix $I_{n_{x}+n_{y}}=$ $\left[\begin{array}{llll}e_{1} & e_{2} & \ldots & e_{n_{x}+n_{y}}\end{array}\right]$. This permits to write $\omega_{k}^{\top} e_{i} e_{i}^{\top} \omega_{k} \leq$ $1, \quad i=1, \ldots, n_{x}+n_{y}$. Then, denoting by $T_{i}=e_{i} e_{i}^{\top}$ the 
matrix having only the element $(i, i)$ equal to 1 , it leads to the scalar inequalities $\omega_{k}^{\top} T_{i} \omega_{k} \leq 1, \quad i=1, \ldots, n_{x}+n_{y}$. This result is further used by the following property.

Property 1: Consider a positive definite matrix $S \in$ $\mathbb{R}^{\left(n_{x}+n_{y}\right) \times\left(n_{x}+n_{y}\right)}$ and the positive real scalars $\rho>0$ and $\tau_{i} \geq 0, \quad i=1, \ldots, n_{x}+n_{y}$. If the conditions (4) are verified:

$$
\left\{\begin{array}{l}
\omega_{k}^{\top} T_{i} \omega_{k} \leq 1, \quad i=1, \ldots, n_{x}+n_{y}, \\
\sum_{\substack{n_{x}+n_{y} \\
n_{x}+n_{y}}} \tau_{i}<\rho, \quad i=1, \ldots, n_{x}+n_{y}, \\
\sum_{i=0} \tau_{i} T_{i} \succ S, \quad \text { with } S \succ 0,
\end{array}\right.
$$

then the following inequality holds $\frac{1}{\rho} \omega_{k} \omega_{k}^{\top} \prec S^{-1}$.

Proof: From (4.b), it is trivial to see that $\rho>\sum_{i=0}^{n_{x}+n_{y}} \tau_{i} \geq$ 0. Multiplying left and right the expression (4.c) by $\omega_{k}^{\top}$ and $\omega_{k}$, respectively, and then successively using (4.a) and (4.c), leads to the following scalar formulation $\omega_{k}^{\top} S \omega_{k}<$ $\omega_{k}^{\top}\left(\sum_{i=0}^{n_{x}+n_{y}} \tau_{i} T_{i}\right) \omega_{k}=\sum_{i=0}^{n_{x}+n_{y}} \tau_{i}\left(\omega_{k}^{\top} T_{i} \omega_{k}\right) \leq \sum_{i=0}^{n_{x}+n_{y}} \tau_{i}<$ $\rho$. This can be rewritten as $\rho-\omega_{k}^{\top} S \omega_{k}>0$, with $S \succ 0$ and further reformulated using the Schur complement: $\left[\begin{array}{cc}\rho & \omega_{k}^{\top} \\ \omega_{k} & S^{-1}\end{array}\right] \succ 0, \quad S \succ 0$ or equivalently $\left[\begin{array}{cc}S^{-1} & \omega_{k} \\ \omega_{k}^{\top} & \rho\end{array}\right] \succ 0, \quad \rho>0$. Applying again the Schur complement [26] gives $S^{-1}-\omega_{k} \rho^{-1} \omega_{k}^{\top} \succ 0, \quad \rho>0$, which leads to $\frac{1}{\rho} \omega_{k} \omega_{k}^{\top} \prec S^{-1}$.

Finding a guaranteed ellipsoid which contains the state vector $x_{k}$ at each sample time $k$ is formulated by the following theorem.

Theorem 1: Consider an initial state vector $x_{0}$ and at sample time $k$ assume that $x_{k} \in \mathcal{E}\left(P, \bar{x}_{k}, \rho_{k}\right)$, with $P=$ $P^{\top} \succ 0$ and $\rho_{k}>0$. If there exist a matrix $Y_{k} \in \mathbb{R}^{n_{x} \times n_{y}}$, a matrix $S=S^{\top} \succ 0$ in $\mathbb{R}^{\left(n_{x}+n_{y}+n_{\delta}\right) \times\left(n_{x}+n_{y}+n_{\delta}\right)}$ and the scalars $\rho_{k+1}>0$ and $\beta \in(0,1)$ for which the following LMI holds for all $A_{\delta} \in \mathcal{V}_{[A]}$ and $C_{\delta} \in \mathcal{V}_{[C]}$ :

$\min _{\beta, Y_{k}, S, \rho_{k+1}} \rho_{k+1}$

subject to

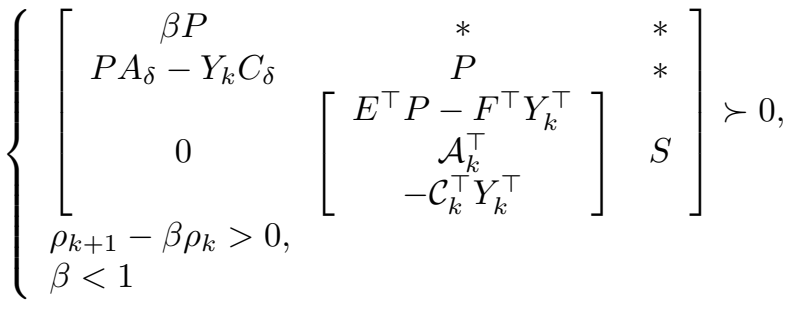

with $*$ denoting the terms required for the symmetry of the matrix and $\mathcal{A}_{k}=\left[\begin{array}{lll}P A_{\delta_{1}} \bar{x}_{k} & \ldots & P A_{\delta_{n_{A_{\delta}}}} \bar{x}_{k}\end{array}\right]$ and $\mathcal{C}_{k}=\left[\begin{array}{lll}C_{\delta_{1}} \bar{x}_{k} & \ldots & C_{\delta_{n C_{\delta}}} \bar{x}_{k}\end{array}\right]$, then the system state $x_{k+1}$ at time $k+1$ is guaranteed to belong to the ellipsoid $\mathcal{E}\left(P, \bar{x}_{k+1}, \rho_{k+1}\right), \forall \omega_{k} \in \mathbf{B}^{n_{x}+n_{y}+n_{\delta}}, \forall A_{\delta} \in[A]$ and $\forall C_{\delta} \in$ $[C]$, with the following notations:

$$
\begin{aligned}
Y_{k} & =P L_{k}, \\
\bar{x}_{k+1} & =\operatorname{mid}[A] \bar{x}_{k}+L_{k}\left(y_{k}-\operatorname{mid}[C] \bar{x}_{k}\right)
\end{aligned}
$$

Remark 1: The gain $L_{k}$ is computed online at each sample time. This improves the convergence of the estimation compared to the technique proposed by [14] which considers a fixed gain.

Proof: Denote by $z_{k}=x_{k}-\bar{x}_{k}$ the error between the real state and the nominal estimated state at time $k$. At time instant $k+1$, the error $z_{k+1}$ is given by $z_{k+1}=x_{k+1}-\bar{x}_{k+1}$. After regrouping the terms in $x_{k}, \omega_{k}$ and $\bar{x}_{k}$ and replacing $\operatorname{mid}[A]$ and $\operatorname{mid}[C]$ as detailed in equations (2) gives:

$$
z_{k+1}=A_{L_{k}} z_{k}+\eta_{L_{k}}+\left(\Delta A_{\delta}-L_{k} \Delta C_{\delta}\right) \bar{x}_{k},
$$

with $A_{L_{k}}=A_{\delta}-L_{k} C_{\delta}$ and $\eta_{L_{k}}=\left(E-L_{k} F\right) \omega_{k}$.

In order to verify the result, the next step is to prove the following expression:

$$
z_{k}^{\top} P z_{k} \leq \rho_{k} \Rightarrow z_{k+1}^{\top} P z_{k+1} \leq \rho_{k+1} .
$$

Consider $F_{0}\left(z_{k}\right)=\rho_{k+1}-\left(A_{L_{k}} z_{k}+\eta_{L_{k}}+\left(\Delta A_{\delta}-\right.\right.$ $\left.\left.L_{k} \Delta C_{\delta}\right) \bar{x}_{k}\right)^{\top} P\left(A_{L_{k}} z_{k}+\eta_{L_{k}}+\left(\Delta A_{\delta}-L_{k} \Delta C_{\delta}\right) \bar{x}_{k}\right)=$ $\rho_{k+1}-z_{k+1}^{\top} P z_{k+1}$ and $F_{1}\left(z_{k}\right)=\rho_{k}-z_{k}^{\top} P z_{k}$. Using the SProcedure defined in [26], expression (9) is verified if there exists $\beta>0$ such that $F_{0}\left(z_{k}\right)-\beta F_{1}\left(z_{k}\right) \geq 0, \quad \forall \omega_{k} \in$ $\mathbf{B}^{n_{x}+n_{y}}, \forall A_{\delta} \in[A]$ and $\forall C_{\delta} \in[C]$, which is equivalent to:

$$
z_{k+1}^{\top} P z_{k+1}+\beta\left(\rho_{k}-z_{k}^{\top} P z_{k}\right) \leq \rho_{k+1} .
$$

Using (8), this is further equivalent to $z_{k}^{\top} A_{L_{k}}^{\top} P A_{L_{k}} z_{k}+$ $\left(\eta_{L_{k}}+\left(\Delta A_{\delta}-L_{k} \Delta C_{\delta}\right) \bar{x}_{k}\right)^{\top} P\left(\eta_{L_{k}}+\left(\Delta A_{\delta}-L_{k} \Delta C_{\delta}\right) \bar{x}_{k}\right)+$ $2\left(\eta_{L_{k}}+\left(\Delta A_{\delta}-L_{k} \Delta C_{\delta}\right) \bar{x}_{k}\right)^{\top} P A_{L_{k}} z_{k}-\beta z_{k}^{\top} P z_{k}-\rho_{k+1}+$ $\beta \rho_{k} \leq 0, \quad \forall \omega_{k} \in \mathbf{B}^{n_{x}+n_{y}}, \forall A_{\delta} \in[A]$ and $\forall C_{\delta} \in[C]$ which can be rewritten as:

$\left[\begin{array}{c}z_{k} \\ 1\end{array}\right]^{\top}\left[\begin{array}{cc}A_{L_{k}}^{\top} P A_{L_{k}}-\beta P & * \\ \left(\eta_{L_{k}}+\left(\Delta A_{\delta}-L_{k} \Delta C_{\delta}\right) \bar{x}_{k}\right)^{\top} P A_{L_{k}} & \gamma\end{array}\right]\left[\begin{array}{c}z_{k} \\ 1\end{array}\right] \prec 0$,

with $\gamma=-\rho_{k+1}+\beta \rho_{k}+\left(\eta_{L_{k}}+\left(\Delta A_{\delta}-\right.\right.$ $\left.\left.L_{k} \Delta C_{\delta}\right) \bar{x}_{k}\right)^{\top} P\left(\eta_{L_{k}}+\left(\Delta A_{\delta}-L_{k} \Delta C_{\delta}\right) \bar{x}_{k}\right), \quad \forall \omega_{k} \in$ $\mathbf{B}^{n_{x}+n_{y}}, \forall A_{\delta} \in[A], \forall C_{\delta} \in[C]$ and $\forall z_{k} \in \mathbb{R}^{n_{x}}$. The expression (10) is verified, $\forall z_{k} \in \mathbb{R}^{n_{x}}$, if:

$$
\left[\begin{array}{cc}
-A_{L_{k}}^{\top} P A_{L_{k}}+\beta P & * \\
-\left(\eta_{L_{k}}+\left(\Delta A_{\delta}-L_{k} \Delta C_{\delta}\right) \bar{x}_{k}\right)^{\top} P A_{L_{k}} & -\gamma
\end{array}\right] \succ 0,
$$

$\forall \omega_{k} \in \mathbf{B}^{n_{x}+n_{y}}, \forall A_{\delta} \in[A]$ and $\forall C_{\delta} \in[C]$, or equivalently:

$$
\left[\begin{array}{cc}
\beta P & 0 \\
0 & \rho_{k+1}-\beta \rho_{k}
\end{array}\right]-\tilde{A}^{\top} P^{-1} \tilde{A} \succ 0,
$$

$\forall \omega_{k} \in \mathbf{B}^{n_{x}+n_{y}}, \forall A_{\delta} \in[A]$ and $\forall C_{\delta} \in[C]$, with $\tilde{A}=\left[\begin{array}{ll}P A_{L_{k}} & P\left(\eta_{L_{k}}+\left(\Delta A_{\delta}-L_{k} \Delta C_{\delta}\right) \bar{x}_{k}\right)\end{array}\right]$. Applying the Schur complement [26] leads to:

$$
\left[\begin{array}{ccc}
\beta P & * & * \\
0 & \rho_{k+1}-\beta \rho_{k} & * \\
P A_{L_{k}} & P\left(\eta_{L_{k}}+\left(\Delta A_{\delta}-L_{k} \Delta C_{\delta}\right) \bar{x}_{k}\right) & P
\end{array}\right] \succ 0
$$

$\forall \omega_{k} \in \mathbf{B}^{n_{x}+n_{y}}, \forall A_{\delta} \in[A], \forall C_{\delta} \in[C]$.

Using the explicit formulation of $A_{L_{k}}, \eta_{L_{k}}$ and $Y_{k}$, the equivalent expression follows:

$$
\left[\begin{array}{ccc}
\beta P & * & * \\
0 & \rho_{k+1}-\beta \rho_{k} & * \\
P A_{\delta}-Y_{k} C_{\delta} & v & P
\end{array}\right] \succ 0
$$


$\forall \omega_{k} \in \mathbf{B}^{n_{x}+n_{y}}, \forall A_{\delta} \in[A], \forall C_{\delta} \in[C]$, with $v=(P E-$ $\left.Y_{k} F\right) \omega_{k}+P\left(\Delta A_{\delta}-L_{k} \Delta C_{\delta}\right) \bar{x}_{k}$.

Pre-multiplying and post multiplying $\Delta A_{\delta}$ in (3) by $P$ and $\bar{x}_{k}$ gives: $P \Delta A_{\delta} \bar{x}_{k}=P \sum_{i=1}^{n_{A_{\delta}}} A_{\delta_{i}} \delta_{i} \bar{x}_{k}=\sum_{i=1}^{n_{A_{\delta}}} P A_{\delta_{i}} \bar{x}_{k} \delta_{i}$. This expression is equivalent to:

$$
P \Delta A_{\delta} \bar{x}_{k}=\mathcal{A}_{k} \delta_{A}
$$

with $\mathcal{A}_{k}=\left[\begin{array}{lll}P A_{\delta_{1}} \bar{x}_{k} & \ldots & P A_{\delta_{n_{A_{\delta}}}} \bar{x}_{k}\end{array}\right] \in \mathbb{R}^{n_{x} \times n_{A_{\delta}}}$ and $\delta_{A}=\left[\begin{array}{lll}\delta_{1} & \ldots & \delta_{n_{A_{\delta}}}\end{array}\right]^{\top} \in \mathbb{R}^{n_{A_{\delta}}}$.

In a similar way, post-multiplying $\Delta C_{\delta}$ in (3) by $\bar{x}_{k}$ gives $\Delta C_{\delta} \bar{x}_{k}=\sum_{i=1}^{n_{C_{\delta}}} C_{\delta_{i}} \delta_{i} \bar{x}_{k}=\sum_{i=1}^{n_{C_{\delta}}} C_{\delta_{i}} \bar{x}_{k} \delta_{i}$, equivalent to:

$$
\Delta C_{\delta} \bar{x}_{k}=\mathcal{C}_{k} \delta_{C}
$$

with $\mathcal{C}_{k}=\left[\begin{array}{lll}C_{\delta_{1}} \bar{x}_{k} & \ldots & C_{\delta_{n}} \bar{x}_{k}\end{array}\right] \in \mathbb{R}^{n_{y} \times n_{C_{\delta}}} \quad$ and $\delta_{C}=\left[\begin{array}{lll}\delta_{1} & \ldots & \delta_{n_{C_{\delta}}}\end{array}\right]^{\top} \in \mathbb{R}^{n_{C_{\delta}}}$.

Replacing (12) and (13) in (11) gives:

$\left[\begin{array}{ccc}\beta P & * & * \\ 0 & \rho_{k+1}-\beta \rho_{k} & * \\ P A_{\delta}-Y_{k} C_{\delta} & \varsigma & P\end{array}\right] \succ \quad 0, \forall \omega_{k} \in$ $\mathbf{B}^{n_{x}+n_{y}}, \quad \delta_{A} \in \mathbf{B}^{n_{A_{\delta}}}, \quad \delta_{C} \in \mathbf{B}^{n_{C_{\delta}}}, \quad \forall A_{\delta} \in[A], \quad \forall C_{\delta} \in$ $[C]$, with $\varsigma=\left(P E-Y_{k} F\right) \omega_{k}+\mathcal{A}_{k} \delta_{A}-Y_{k} \mathcal{C}_{k} \delta_{C}$, or equivalently:

$$
\left[\begin{array}{ccc}
\beta P & * & * \\
0 & \rho_{k+1}-\beta \rho_{k} & * \\
P A_{\delta}-Y_{k} C_{\delta} & G_{k} r & P
\end{array}\right] \succ 0
$$

$\forall A_{\delta} \in[A], \quad \forall C_{\delta} \in[C], \quad \forall r \in \mathbf{B}^{n_{x}+n_{y}+n_{\delta}}$, with $G_{k}=\left[\begin{array}{lll}P E-Y_{k} F & \mathcal{A}_{k} & -Y_{k} \mathcal{C}_{k}\end{array}\right] \in \mathbb{R}^{n_{x} \times\left(n_{x}+n_{\delta}\right)}$ and $r=\left[\begin{array}{lll}\omega_{k}^{\top} & \delta_{A}^{\top} & \delta_{C}^{\top}\end{array}\right]^{\top}$.

Pre-multiplying and post multiplying inequality by

$$
\left[\begin{array}{lll}
I & 0 & 0 \\
0 & 0 & I \\
0 & I & 0
\end{array}\right]
$$

leads

$$
\begin{aligned}
& {\left[\begin{array}{ccc}
\beta P & * & * \\
P A_{\delta}-Y_{k} C_{\delta} & P & * \\
0 & r^{\top} G_{k}^{\top} & \rho_{k+1}-\beta \rho_{k}
\end{array}\right] \succ 0,} \\
& \forall r \in \mathbf{B}^{n_{x}+n_{y}+n_{\delta}} \\
& \text { with } \rho_{k+1}-\beta \rho_{k}>0 .
\end{aligned}
$$

Applying the Schur complement gives:

$$
\left[\begin{array}{cc}
\beta P & * \\
P A_{\delta}-Y_{k} C_{\delta} & P-G_{k} r \frac{1}{\rho_{k+1}-\beta \rho_{k}} r^{\top} G_{k}^{\top}
\end{array}\right] \succ 0,
$$

$\forall r \in \mathbf{B}^{n_{x}+n_{y}+n_{\delta}}$ with $\rho_{k+1}-\beta \rho_{k}>0$. Applying Property 1 to the term $r \frac{1}{\rho_{k+1}-\beta \rho_{k}} r^{\top}$, with $\rho=\rho_{k+1}-\beta \rho_{k}$, means that $\exists S=S^{\top} \succ 0$ such that $r \frac{1}{\rho_{k+1}-\beta \rho_{k}} r^{\top} \prec S^{-1}$ or equivalently $-r \frac{1}{\rho_{k+1}-\beta \rho_{k}} r^{\top} \succ-S^{-1}, \quad \rho_{k+1}-\beta \rho_{k}>0$. Therefore, the following expression is verified:

$$
\left[\begin{array}{cc}
\beta P & * \\
P A_{\delta}-Y_{k} C_{\delta} & P-G_{k} S^{-1} G_{k}^{\top}
\end{array}\right] \succ 0,
$$

$\forall r \in \mathbf{B}^{n_{x}+n_{y}+n_{\delta}}$, with $S \succ 0$. The constraint (16) can be further decomposed into:

$$
\left[\begin{array}{cc}
\beta P & * \\
P A_{\delta}-Y_{k} C_{\delta} & P
\end{array}\right]-\left[\begin{array}{c}
0 \\
G_{k}
\end{array}\right] S^{-1}\left[\begin{array}{ll}
0 & G_{k}^{\top}
\end{array}\right] \succ 0
$$

with $S \succ 0, \quad \forall r \in \mathbf{B}^{n_{x}+n_{y}+n_{\delta}}$. Applying the Schur Complement and replacing $G_{k}=\left[\begin{array}{lll}P E-Y_{k} F & \mathcal{A}_{k} & -Y_{k} \mathcal{C}_{k}\end{array}\right]$ leads to expression (5). Since $A_{\delta}$ and $C_{\delta}$ appear in an affine way in the LMI (17), the inequality is satisfied if and only if it is verified for all the vertices of $[A]$ and $[C]$.

Given a scalar $\beta \in(0,1)$ in the first step, an initialization is required to set the positive definite matrix $P$.

Remark 2: (Initialisation) Given the rate of the estimation convergence $\beta \in(0,1)$, the center $\bar{x}_{0}$ and the radius $\rho_{0}$ of the initial ellipsoid, the matrix $P$ is computed off-line in the first step (i.e. $k=0$ ) by solving the following optimization problem:

$$
\begin{aligned}
& \min _{P, S, Y_{0}, \rho_{1}} \rho_{1} \\
& \text { subject to } \\
& {\left[\begin{array}{ccc}
\beta P & * & * \\
P A_{\delta}-Y_{0} C & P & * \\
0 & {\left[\begin{array}{c}
E^{\top} P-F^{\top} Y_{0}^{\top} \\
\mathcal{A}_{0}^{\top} \\
-\mathcal{C}_{0}^{\top} Y_{0}^{\top}
\end{array}\right]}
\end{array}\right] \succ 0,}
\end{aligned}
$$

$\rho_{1}-\beta \rho_{0}>0$,

where $\mathcal{A}_{0}=\left[\begin{array}{lll}P A_{\delta_{1}} \bar{x}_{0} & \ldots & P A_{\delta_{n_{\delta}}} \bar{x}_{0}\end{array}\right], \forall A_{\delta} \in[A]$, $\forall C_{\delta} \in[C]$ with:

$$
\begin{aligned}
& Y_{0}=P L_{0}, \\
& \bar{x}_{1}=\operatorname{mid}[A] \bar{x}_{0}+L_{0}\left(y_{0}-\operatorname{mid}[C] \bar{x}_{0}\right)
\end{aligned}
$$

with $y_{0}$ the initial measurement. This initialization sets the matrix $P$.

Remark 3: The number of scalar decision variables in the problem (5) is equal to $2+n_{x} n_{y}+\left(n_{x}+n_{y}+n_{\delta}\right)^{2}$ and equal to $1+\frac{n_{x} \times\left(n_{x}-1\right)}{2}+n_{x} n_{y}+\left(n_{x}+n_{y}+n_{\delta}\right)^{2}$ for the initialization problem (18).

Remark 4: (Vertex reduction) The LMI problem (5) has to be verified in $2^{n_{\delta}}$ vertices. In the general case when all the elements of the matrices $A_{\delta}$ and $C_{\delta}$ have interval uncertainties, the value of $n_{\delta}$ is equal to $n_{x}^{2}+n_{x} n_{y}$. In order to reduce the number of vertices to be verified from $2^{n_{\delta}}$ to $2^{4 n_{x}}$ the following optimization problem can be solved (applying the scaling technique of matrices of Theorem 1 of [30]):

$$
\begin{aligned}
& \min _{\beta, Y_{k}, S, \rho_{k+1}} \rho_{k+1} \\
& \text { subject to }
\end{aligned}
$$

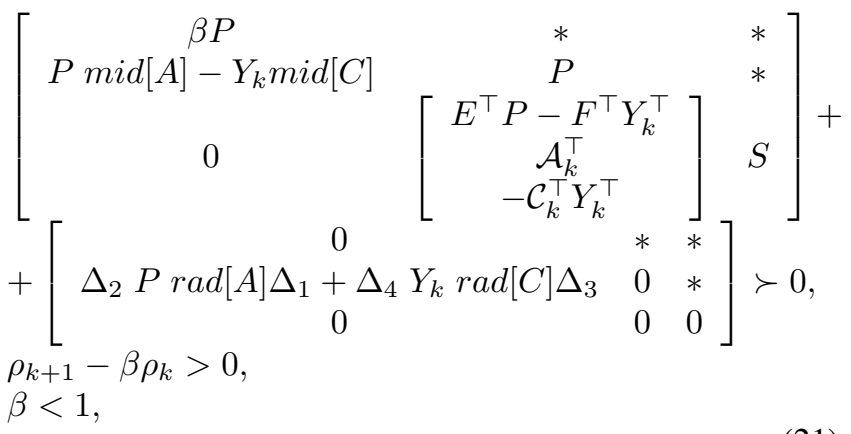

$\forall \Delta_{1} \in \boldsymbol{\Delta}_{n_{x}}, \forall \Delta_{2} \in \boldsymbol{\Delta}_{n_{x}}, \forall \Delta_{3} \in \boldsymbol{\Delta}_{n_{x}}$ and $\forall \Delta_{4} \in \boldsymbol{\Delta}_{n_{x}}$, with $\boldsymbol{\Delta}_{n_{x}}$ the set of $n_{x} \times n_{x}$ diagonal matrices with diagonal entries equal to 1 or -1 . 


\section{ILLUSTRATIVE EXAMPLE}

Consider the linear discrete-time invariant system (1) with $A=\left[\begin{array}{ll}0.7+0.3 \delta_{1} & 0.1+0.1 \delta_{2} \\ 0.6+0.1 \delta_{3} & 0.2+0.1 \delta_{4}\end{array}\right], C=$ $\left[\begin{array}{cc}-2+0.1 \delta_{5} & 1 \\ 1 & 1+0.1 \delta_{6}\end{array}\right], E=\left[\begin{array}{cccc}0.05 & 0 & 0 & 0 \\ 0 & 0.02 & 0 & 0\end{array}\right]$, $F=\left[\begin{array}{cccc}0 & 0 & 0.05 & 0 \\ 0 & 0 & 0 & 0.05\end{array}\right],\left\|\omega_{k}\right\|_{\infty} \leq 1,\left|\delta_{i}\right|<1, i=$ $1, \ldots, 6$. The value of $\omega_{k}$ is randomly generated. The initial state belongs to the ellipsoid $\mathcal{E}\left(P_{0}, \bar{x}_{0}, \rho_{0}\right)$ with $P_{0}=I_{2}$, $\bar{x}_{0}=\left[\begin{array}{ll}0 & 0\end{array}\right]^{\top}$ and $\rho_{0}=1$ which is sufficiently large to contain the initial state. In this example, the results obtained by the proposed approach (5) is compared in terms of accuracy and complexity to the results obtained by the off-line $P$-radiusbased zonotopic state estimation [14], [31].

Remark 5: Notice that the $P$-radius zonotopic estimation method is developed in the case of a constant known observation matrix $C$ (only the evolution matrix $A_{\delta}$ is uncertain). To summarize, an interval matrix $C_{\delta}$ is used with the ellipsoidal estimation method (5), while a fixed matrix $C$ is used with the zonotopic estimation [14], [31].

Figures 1 and 2 illustrate the bounds of $x_{1}$ and $x_{2}$ after 50 iterations obtained by the online ellipsoidal and the off-line zonotopic estimations.

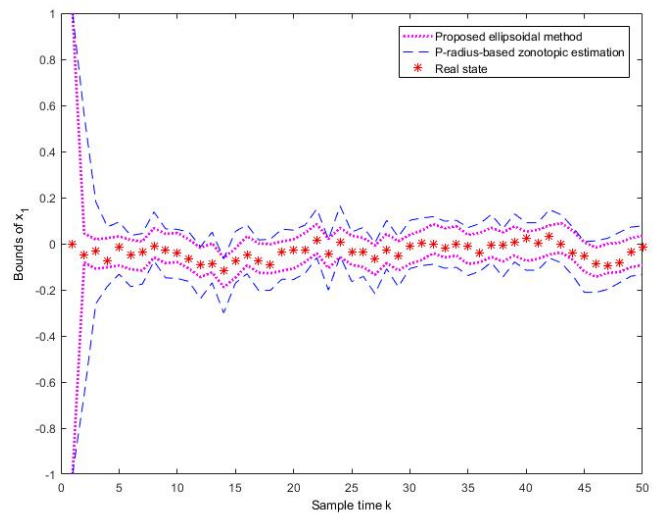

Fig. 1. Bounds of $x_{1}$

The blue dashed lines are obtained by the zonotopic estimation based on the zontope $P$-radius minimization, the magenta dotted lines are obtained using the ellipsoidal estimation based on the minimization of the ellipsoid radius (5). The red stars, representing the real state of the system are situated inside the estimated bounds, which validates the guaranteed estimation.

In order to facilitate the comparison between these methods, Fig. 3 and Fig. 4 illustrate the bounds widths of $x_{1}$ and $x_{2}$, respectively. The off-line zonotopic $P$-radius based method is considered as reference. The better accuracy of the estimation is obtained using the online ellipsoidal method.

Figure 5 compares the volume of the state estimation sets. The volume obtained by the ellipsoidal estimation is less

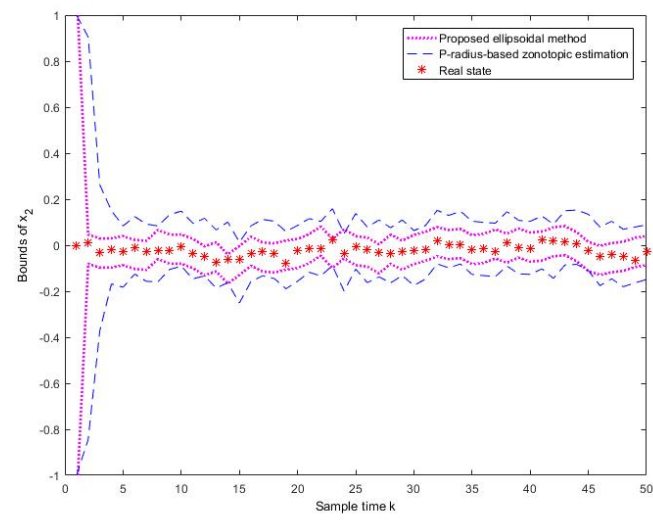

Fig. 2. Bounds of $x_{2}$

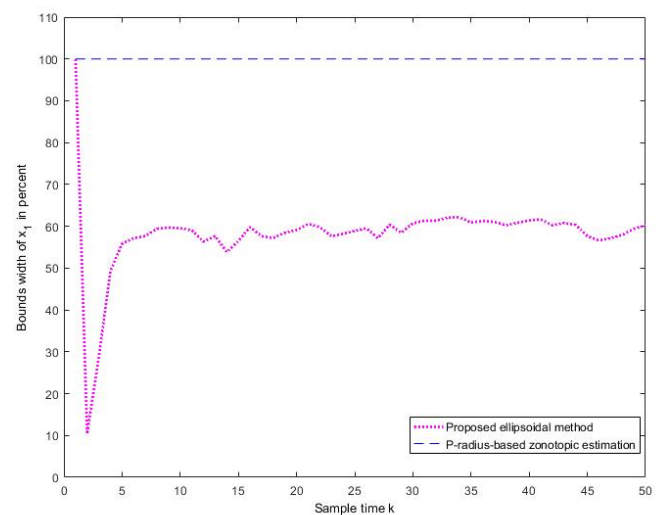

Fig. 3. Comparison of the bounds width of $x_{1}$

than the volume of the estimation set obtained by the $P$ radius-based zonotopic estimation method.

The proposed methods offer a good accuracy compared to the $P$-radius-based zonotopic estimation [31] but with increased complexity due to the online computation of the ellipsoidal radius. The computation time of one iteration is equal to $0.1 \mathrm{~s}$ for the ellipsoidal method (5) considering interval uncertainties both in the $A$ and $C$ matrices, while equal to $0.01 \mathrm{~s}$ using the off-line $P$-radius-based zonotopic estimation considering interval uncertainties only in the $A$ matrix.

The proposed online ellipsoidal estimation method gives a better estimation (despite considering additional interval uncertainties on the $C$ matrix) than the estimation obtained by the off-line $P$-radius zonotopic estimation due to the minimization (with a known $C$ matrix).

\section{CONClusion}

A new approach for guaranteed ellipsoidal state estimation for multivariable linear discrete-time invariant systems with interval uncertainties (in both the evolution and observation matrices) and bounded perturbations and measurement noises 


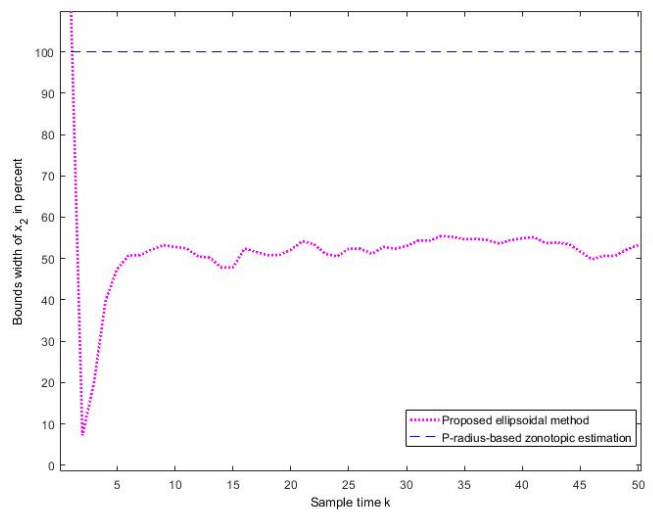

Fig. 4. Comparison of the bounds width of $x_{2}$

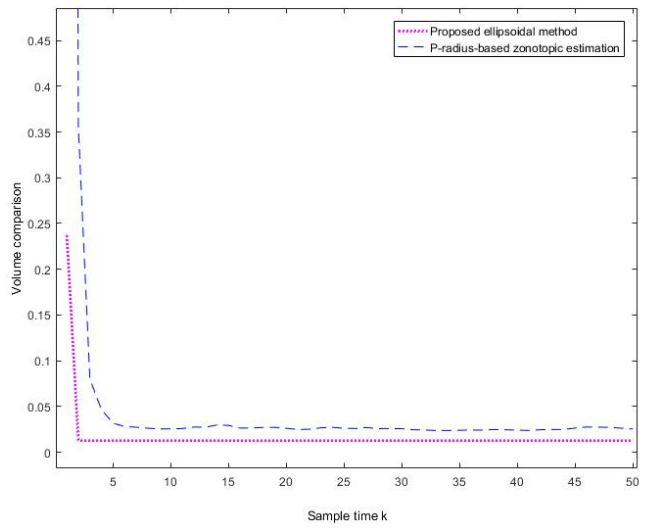

Fig. 5. Comparison of the volume of the state estimation sets

has been proposed. This approach is based on the online minimization of the ellipsoidal radius of the state estimation set by solving a Linear Matrix Inequality optimization problem. This method offers an estimation less conservative than the estimation obtained by the $P$-radius zonotopic estimation but with increased complexity due to the online computation of the ellipsoidal radius. An interesting perspective is to apply this method to fault detection and fault tolerant purposes.

\section{REFERENCES}

[1] R. E. Kalman, "A new approach to linear filtering and prediction problems," Transactions of the ASME-Journal of Basic Engineering, vol. 82, no. Series D, pp. 35-45, 1960.

[2] F. C. Schweppe, "Recursive state estimation: unknown but bounded errors and system inputs," IEEE Transactions on Automatic Control, vol. 13(1), pp. 22-28, 1968.

[3] E. Fogel, "System identification via membership set constraints with energy constrained noise," IEEE Transactions on Automatic Control, vol. 24, p. 752, 1979.

[4] D. P. Bertsekas and I. B. Rhodes, "Recursive state estimation for a set-membership description of uncertainty," IEEE Transactions on Automatic Control, vol. 16(2), pp. 117-128, 1971.

[5] S. H. Witsenhausen, "Sets of possible states of linear systems given perturbed observations," IEEE Transactions on Automatic Control, vol. 13, pp. 556-558, 1968.
[6] F. L. Chernousko and D. Y. Rokityanskii, "Ellipsoidal bounds on reachable sets of dynamical systems with matrices subjected to uncertain perturbations," Journal of Optimization Theory and Applications, vol. 104, pp. 1-19, 2000.

[7] L. Chisci, A. Garulli, and G. Zappa, "Recursive state bounding by parallelotopes," Automatica, vol. 32, pp. 1049-1055, 1996.

[8] F. Mazenc, T. Dinh, and S. Niculescu, "Interval observers for discretetime systems," International Journal of Robust Nonlinear Control, vol. 17, pp. 2867-2890, 2014

[9] P. Massioni, N. Salnikov, and G. Scorletti, "Ellipsoidal state estimation based on sum of squares for nonlinear systems with unknown but bounded noise," IET Control Theory \& Applications, vol. 13, pp. 1955-1961, 2019.

[10] E. Walter and H. Piet-Lahanier, "Exact recursive polyhedral description of the feasible parameter set for bounded-error models," IEEE Transactions on Automatic Control, vol. 34(8), pp. 911-915, 1989.

[11] V. Puig, P. Cugueró, and J. Quevedo, "Worst-case estimation and simulation of uncertain discrete-time systems using zonotopes," in Proc. of Europeen Control Conference, Portugal, 2001.

[12] C. Combastel, "A state bounding observer based on zonotopes," in Proc. of European Control Conference, Cambridge, UK, 2003.

[13] T. Alamo, J. M. Bravo, and E. F. Camacho, "Guaranteed state estimation by zonotopes," Automatica, vol. 41, pp. 1035-1043, 2005.

[14] V. T. H. Le, T. Alamo, E. F. Camacho, C. Stoica, and D. Dumur, Zonotopes: From Guaranteed State Estimation to Control. ISTE Ltd and John Wiley \& Sons, Inc., 2013.

[15] V. T. H. Le, C. Stoica, D. Dumur, T. Alamo, and E. Camacho, "Robust tube-based constrained predictive control via zonotopic setmembership estimation," in Proc. of the 50th IEEE CDC and ECC, 2011, pp. 4580-4585, Orlando, Florida, USA.

[16] V. T. H. Le, T. Alamo, E. F. Camacho, C. Stoica, and D. Dumur, "Zonotopic set-membership estimation for interval dynamic systems," in Proc. of the ACC, 2012, Montreal, QC, Canada.

[17] A. B. Kurzhanski and I. Vályi, Ellipsoidal calculus for estimation and control. Birkhaüser Boston, 1996.

[18] C. Durieu, E. Walter, and B. Polyak, "Multi-input multi-output ellipsoidal state bounding," Journal of optimization theory and applications, vol. 111(2), pp. 273-303, 2001.

[19] B. T. Polyak, S. A. Nazin, C. Durieu, and E. Walter, "Ellipsoidal parameter or state estimation under model uncertainty," Automatica, vol. 40, pp. 1171-1179, 2004

[20] F. L. Chernousko, State Estimation for Dynamic Systems. Boca Raton: CRC Press, 1994

[21] T. $\mathrm{Hu}$ and $\mathrm{Z}$. Lin, "Composite quadratic Lyapunov functions for constrained control systems," IEEE Transaction on Automatic Control, vol. 48, pp. 440-450, 2003.

[22] C. Combastel and S. A. Raka, "A Stable Interval Observer for LTI Systems with No Multiple Poles," in Proc. of the 18th World Congress IFAC, Milano, Italy, 2011.

[23] D. Efimov, W. Perruquetti, T. Raïssi, and A. Zolghadri, "On Interval Observer Design for Time-Invariant Discrete-Time Systems," in Proc. of European Control Conference, Zürich, Switzerland, 2010.

[24] F. Mazenc and O. Bernard, "Interval observers for linear time-invariant systems with disturbances," Automatica, vol. 47, pp. 140-147, 2011.

[25] L. Jaulin, M. Kieffer, O. Didrit, and E. Walter, Applied Interval Analysis. Springer, 2001.

[26] S. Boyd, L. E. Ghaoui, E. Feron, and V. Balakrishnan, Linear Matrix Inequalities in System and Control Theory. Philadelphia: SIAM, 1994.

[27] S. Ben Chabane, C. Stoica Maniu, T. Alamo, E. F. Camacho, and D. Dumur, "A new approach for guaranteed ellipsoidal state estimation," in Proc. of the 19th World Congress IFAC, Cape Town, 2014.

[28] S. Ben Chabane, C. Stoica Maniu, T. Alamo, E. F. Camacho, and D. Dumur, "Ellipsoidal state estimation for systems with interval uncertainties," in Proc. of the 53d IEEE Conference on Decision and Control, 2014, Los Angeles, USA.

[29] Y. Yang, "Controllability and observability of a class of linear, time-invariant systems with interval plants," International Journal of Information and Systems Sciences, vol. 1, pp. 184-192, 2005.

[30] T. Alamo, R. Tempo, D. R. Ramírez, and E. F. Camacho, "A new vertex result for robustness problems with interval matrix uncertainty," Systems and Control Letters, vol. 57, pp. 474-481, 2008.

[31] V. T. H. Le, C. Stoica, T. Alamo, E. F. Camacho, and D. Dumur, "Zonotopic guaranteed state estimation for uncertain systems," Automatica, vol. 49(1), pp. 3418-3424, 2013. 